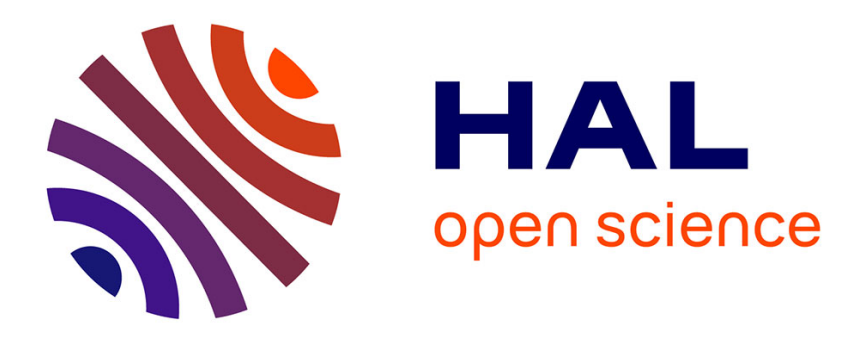

\title{
Critical relaxation in three-dimensional spin glasses
}

\author{
M.V. Feigel'Man, L.B. Ioffe
}

\section{To cite this version:}

M.V. Feigel'Man, L.B. Ioffe. Critical relaxation in three-dimensional spin glasses. Journal de Physique Lettres, 1985, 46 (15), pp.695-702. 10.1051/jphyslet:019850046015069500 . jpa-00232582

\section{HAL Id: jpa-00232582 https://hal.science/jpa-00232582}

Submitted on 1 Jan 1985

HAL is a multi-disciplinary open access archive for the deposit and dissemination of scientific research documents, whether they are published or not. The documents may come from teaching and research institutions in France or abroad, or from public or private research centers.
L'archive ouverte pluridisciplinaire HAL, est destinée au dépôt et à la diffusion de documents scientifiques de niveau recherche, publiés ou non, émanant des établissements d'enseignement et de recherche français ou étrangers, des laboratoires publics ou privés. 
Classification

Physics Abstracts

75.40D

\title{
Critical relaxation in three-dimensional spin glasses
}

\author{
M. V. Feigel'man and L. B. Ioffe \\ L. D. Landau Institute for Theoretical Physics, U.S.S.R. Academy of Sciences, \\ 117940 Moscow V-334, ul. Kosygina 2, U.S.S.R.
}

(Reçu le 19 mars 1985, accepté sous forme définitive le 11 juin 1985)

\begin{abstract}
Résumé. - Nous avons développé la théorie analytique de la relaxation lente des verres de spin 3-D vectoriels et ceux d'Ising avec interaction de portée finie près du point de transition de gélation. Dans le cas d'Ising le temps maximum de relaxation, $t_{\max }$, croît comme $t_{\max } \sim\left(T-T_{\mathrm{f}}\right)^{-6} \exp \left(C\left(T-T_{\mathrm{f}}\right)^{-1 / 4}\right)$. Dans le cas des spins d'Heisenberg $t_{\max } \sim\left(T-T_{\mathrm{f}}\right)^{-10,5}$. Nous montrons que l'inclusion de l'échange d'anisotropie faible aléatoire conduit à un comportement asymptotique d'Ising pour les verres de spin d'Heisenberg.
\end{abstract}

Abstract. - The analytical theory of slow relaxation of the Ising and vector 3-D spin glasses with the finite range interaction near the freezing transition point $T_{\mathrm{f}}$ has been developed. In the Ising case, the maximum relaxation time $t_{\max }$ increases as $t_{\max } \sim\left(T-T_{\mathrm{f}}\right)^{-6} \exp \left[C\left(T-T_{\mathrm{f}}\right)^{-1 / 4}\right]$. For the Heisenberg spins $t_{\max } \sim\left(T-T_{\mathrm{f}}\right)^{-10.5}$. It is shown that the inclusion of weak random-anisotropy exchange leads to the Ising asymptotics for $t_{\max }$ in the Heisenberg spin glass.

1. - We consider the relaxation dynamics of the 3-D spin glass (Edwards-Anderson model [1] with long (but finite) range interaction) slightly above the phase transition point $[2,3]$. The spin interaction energy is

$$
H=-\frac{1}{2} \sum_{i j} j_{i j} \sigma_{i} \sigma_{j}
$$

Summation in this formula is performed over sites $(i)$ with spins $\sigma_{i}$, which we suppose to be either classical $n$-component unit vectors, or (for $n=1$ ) Ising variables. The sites $(i)$ are distributed randomly with concentration $c, \mathfrak{J}_{i j}$ are independent random variables, $\overline{\mathfrak{J}}_{i j}=0, \mathfrak{J}_{i j}^{2}=K\left(\mathbf{r}_{i}-\mathbf{r}_{j}\right)$. The function $K(r)$ obeys the normalization condition

$$
\int K(r) \mathrm{d}^{3} r=1
$$

and the condition of a large number of interacting neighbours :

$$
Z_{0}=c\left[\int K(r) r^{2} \mathrm{~d}^{3} r\right]^{3 / 2} \gg 1
$$

The exact form of $K(r)$ is irrelevant at $Z_{0} \gg 1$. 
We restrict ourselves to the simplest problem of the purely dissipative dynamics, described by the Langevin equations $(n \geqslant 2)$ :

$$
\dot{\sigma}_{i}=-\Gamma_{0} \frac{\delta H}{\delta \sigma_{i}}+\zeta_{i}(t),\left\langle\zeta_{i}^{\alpha}(t) \zeta_{j}^{\beta}\left(t^{\prime}\right)\right\rangle=2 T \Gamma_{0} \delta_{i j} \delta^{\alpha \beta} \delta\left(t-t^{\prime}\right)
$$

where $T$ is the temperature (note that the normalization condition (2) leads to the " mean field" phase transition temperature $T_{0}=1 / n$ ), and $\Gamma_{0}^{-1}$ is the one spin relaxation time. For the case of the Ising spins, instead of equation(4) we shall adopt their physical equivalent for the Ising spins the Glauber kinetic equations :

$$
\begin{aligned}
\dot{p}\left\{\sigma_{i}\right\}=\Gamma_{0} \sum_{j}\left[-\exp \left[-\frac{1}{T} \sum_{k} \mathfrak{J}_{j k} \sigma_{k}\right] p\left\{\sigma_{1} \ldots \sigma_{j} \ldots \sigma_{n}\right\}+\right. \\
+\exp \left[\frac{1}{T} \sum_{k} \mathfrak{J}_{j k} \sigma_{k}\right] p\left\{\sigma_{1} \ldots \sigma_{j} \ldots \sigma_{n}\right\}+
\end{aligned}
$$

where $p\left\{\sigma_{i}\right\}$ is the time-dependent probability of the $\left\{\sigma_{i}\right\}$ spin configuration.

2. - In the proceding Letter [2] we have considered the thermodynamics of the model defined by equations (1) to (3) in the Ising case and proved the existence of a genuine phase transition at $T=T_{\mathrm{f}}\left(T_{0}-T_{\mathrm{f}} \sim Z^{-1 / 3} T_{0}\right)$; (note, that $\left(T_{0}-T_{\mathrm{f}}\right) / T_{0} \gg \tau_{0} \sim Z^{-2 / 3}$, $\tau_{0}$ being the temperature range where mean field approximation does not hold.)

This genuine phase transition cannot be regarded as the condensation into one macroscopic delocalized mode as it can usually be done for an ordinary phase transition, but it rather signals the appearance of an infinite hierarchy of localized modes. Qualitatively this phase transition occurs as follows : as the temperature drops to $\left(T-T_{0}\right) / T_{0} \sim \tau_{0}$, highly correlated spins ( " clusters ») appear (the term " cluster» does not describe these formations well because it usually implies something very rigid whereas here two clusters can overlap; however, we could not find a better term). As the temperature falls, the number of clusters rapidly increases, they begin to overlap strongly and interact with each other : at first only the interaction of the order parameter absolute values, corresponding to different clusters is important, whereas when the temperature is further lowered down to $\left(T_{0}-T\right) / T \sim Z^{-1 / 3}$ the interaction of the signs of the order parameters becomes also important. This sign interaction is equivalent to the initial model (1) to (3) with the renormalized parameters : the effective temperature and the number of neighbours, the latter increasing with a transition to the next hierarchy level $\left(Z_{1} \simeq Z^{4 / 3}\right)$. At a further temperature decrease highly correlated clusters of signs appear repeatedly. At $T=T_{\mathrm{f}}$ the hierarchy of correlations becomes infinite, the whole system at this temperature resembles a hierarchy of superparamagnets where a spin of the next level of the hierarchy is formed by a large number of spins of the previous level. A slightly analogous model for the glass dynamics has been recently proposed a priori in [4]. The qualitative features of the phase transition are the same for the case of the vector spins, but the values of $T_{0}-T_{\mathrm{f}}$ and $Z_{1}$ are slightly different $:\left(T_{0}-T_{\mathrm{f}}\right) \sim T_{0} Z^{-4 / 15}$, $Z_{1} \sim Z^{32 / 15}$.

Let us explain how these results have been obtained. The critical modes must be picked out near the transition point. In our case it means that the averaging over fast fluctuations must be carried out and then the variables $\mathbf{a}_{\lambda}$ must be introduced instead of the variables $\mathbf{m}_{i}=\left\langle\sigma_{i}\right\rangle_{\mathrm{f}}$ (where \langle\rangle$_{\mathrm{f}}$ denotes the average over " fast » fluctuations) these new variables can be defined as the expansion of $\mathbf{m}_{i}$ over eigenfunctions $\psi_{\lambda}(i)$ of the $\mathfrak{J}_{i j}$ - matrix : $\mathbf{m}_{i}=\sum_{\lambda} \mathbf{a}_{\lambda} \psi_{\lambda}(i), \sum_{i} \mathfrak{J}_{j i} \psi_{\lambda}(i)=$ $\psi_{\lambda}(j) E_{\lambda}$. The variables $\mathbf{a}_{\lambda}$, corresponding to the eigenfunctions $\psi_{\lambda}(i)$ with eigenvalues $\left|E_{\lambda}-2\right| \lesssim$ $Z^{-4 / 3}$ (i.e. eigenvalues near the spectrum boundary) are the sought for critical modes. The respective eigenfunctions $\psi_{\lambda}(i)$ are localized, they serve as clusters, discussed in the previous paragraph. 
To obtain the quantitative results we have used the following hypothesis from the localization theory about the eigenfunctions of the $j_{i j}$-matrix :

$$
\overline{\left[\sum \psi_{\lambda}^{4}(i)\right]^{-1}} \sim l^{d}\left(\varepsilon_{\lambda}\right) \sim\left(\varepsilon-\varepsilon_{\mathrm{c}}\right)^{-d v}
$$

where $d$ is the space dimension, $l$ is the localization length, $\varepsilon_{\mathrm{c}}$ is the mobility edge and $v=(d-2)^{-1}$ is the critical exponent.

3. - Now we consider the critical dynamics near $T_{0}\left(\tau=\left(T-T_{0}\right) / T_{0} \ll 1\right)$. In this temperature range the relaxation is slow : $t \gtrsim \tau^{-1} \Gamma_{0}^{-1}$, therefore the averaging over fast fluctuations (with $t \ll \tau^{-1} \Gamma_{0}^{-1}$ ) can be carried out. These fast fluctuations are well described by the mean field approximation. The averaging yields the dynamic Langevin equations for $\mathbf{m}_{i}=\left\langle\sigma_{i}\right\rangle_{\mathrm{f}}$ :

$$
\dot{\mathbf{m}}_{i}=-\Gamma_{0} \frac{\delta H_{\mathrm{TAP}}}{\delta \mathrm{m}_{i}}+\zeta_{i}(t)
$$

where $H_{\text {TAP }}$ coincides with the free energy obtained in papers [5, 6]. The small corrections (of the order of $m_{i}^{2}$ ) to the $\Gamma_{0}$ coefficient are neglected :

$$
\begin{aligned}
H_{\mathrm{TAP}}=-\frac{1}{2} \sum_{i j} \mathfrak{J}_{i j} \mathbf{m}_{i} \mathbf{m}_{j}-\frac{1}{4 n T} J_{i j}^{2}\left(1-\mathbf{m}_{i}^{2}\right)\left(1-\mathbf{m}_{j}^{2}\right)+ \\
\quad \hbar n T \sum_{i}\left[\frac{\mathbf{m}_{i}^{2}}{2}+\frac{n}{4(n+2)}\left(\mathbf{m}_{i}^{2}\right)^{2}+\frac{1}{6} \frac{n^{2}}{n+4} \frac{n+8}{(n+2)^{2}}\left(\mathbf{m}_{i}^{2}\right)^{3}\right]
\end{aligned}
$$

Next we expand $\mathbf{m}_{i}$ over eigenfunctions of the $\mathfrak{J}_{i j}$-matrix, and single out the eigenfunctions near the $\mathfrak{J}_{i j}$-spectrum boundary :

$$
\begin{aligned}
& \mathbf{m}_{i}=\sum_{\lambda} \mathbf{a}_{\lambda} \psi_{\lambda}(i)+\sum_{\mu} \mathbf{b}_{\mu} \psi_{\mu}(i), \\
& \left|\varepsilon_{\lambda}-2\right| \leqslant \alpha,\left|\varepsilon_{\mu}-2\right|>\alpha
\end{aligned}
$$

where $\alpha$ is an arbitrary parameter, $Z^{-4 / 3} \ll \alpha \ll 1$. The slow relaxation of the $a_{\lambda}$ variables induces the slow relaxation of the $\mathbf{b}_{\mu}$ variables due to the mode interaction, however the $\mathbf{b}_{\boldsymbol{\mu}}$ variables relaxation to their induced values is fast, hence they can be excluded from equations (6) and (7) which yield :

$$
\begin{aligned}
\dot{\mathbf{a}}_{\lambda} & =-\Gamma_{0} \frac{\delta \tilde{H}}{\delta \mathbf{a}_{\lambda}}+\zeta(t),\left\langle\zeta_{\lambda}^{\alpha}(t) \zeta_{\lambda^{\prime}}^{\beta}\left(t^{\prime}\right)\right\rangle=2 \Gamma_{0} T \delta_{\lambda \lambda^{\prime}} \delta^{\alpha \beta} \delta\left(t-t^{\prime}\right) \\
\tilde{H}\left\{\mathbf{a}_{\lambda}\right\} & =-\frac{1}{2} \sum_{i j} j_{i j} \tilde{\mathbf{m}}_{i} \tilde{\mathbf{m}}_{j}-\frac{1}{4 n T} \sum_{i j} j_{i j}^{2}\left(1-\tilde{\mathbf{m}}_{i}^{2}\right)\left(1-\tilde{\mathbf{m}}_{j}^{2}\right)+T \sum_{i}\left\{\frac{\tilde{\mathbf{m}}_{i}^{2}}{2}+\right. \\
+ & \left.\frac{n}{4(n+2)}\left(\tilde{\mathbf{m}}_{i}^{2}\right)^{2}+\frac{1}{6} \frac{n^{2}}{n+4} \frac{n+8}{(n+2)^{2}}\left(\tilde{\mathbf{m}}_{i}^{2}\right)^{3}\right\}-\frac{1}{2}\left[\frac{T n^{2}}{n+2}\right]^{2} \sum_{i j} \tilde{\mathbf{m}}_{i}^{2} \tilde{\mathbf{m}}_{i} g_{i j} \tilde{\mathbf{m}}_{j}^{2} \tilde{\mathbf{m}}_{j}
\end{aligned}
$$

where $\tilde{\mathbf{m}}_{i}=\sum_{\lambda} \mathbf{a}_{\lambda} \psi_{\lambda}(i), g_{i j}=\sum_{\mu} \psi_{\mu}(i) \psi_{\mu}(j) \theta\left(2-E_{\mu}-\alpha\right) /\left(2-E_{\mu}\right)$. Note that this procedure has been tacitly used by D. J. Thouless, P. W. Anderson and R. G. Palmer when they have investigated the equilibrium phase transition [5] at $Z=\infty$. The results of the mean field theory can be obtained from the free energy $\tilde{H}_{\text {TAP }}$ in the limit $Z \rightarrow \infty$ in the vicinity of the phase transition point.

The coefficient of term quadratic over $\mathbf{a}_{\lambda}$ in $\tilde{H}_{\mathrm{TAP}}$ is equal to $\left(-E_{\lambda}+\left(n T+(n T)^{-1}\right)\right) / 2 \approx$ $\left(-E_{\lambda}+2+\tau^{2}\right) / 2$ therefore the more the temperature decreases $(\tau \rightarrow 0)$, the larger the number 
of modes which become unstable in the quadratic approximation (but, certainly, these modes regain their stability if higher order terms are taken into account). We shall call these modes " filled ". At $\tau \sim Z^{-2 / 3}$ the filled modes begin overlapping and their interaction must be taken into account. At not too low temperatures $\left(|\tau| \gtrsim Z^{-1 / 3}\right.$ for $n=1,|\tau| \gtrsim Z^{-4 / 15}$ for $\left.n \geqslant 2\right)$. Only the leading terms of the interaction, i.e. the terms even with respect to each $\mathbf{a}_{\lambda}$, can be retained. Note that this interaction couples only $\mathbf{a}_{\lambda}^{2}$, hence the signs or the directions of the spins fluctuate freely. At $-\tau \gg Z^{-2 / 3}$ the number of overlapping eigenfunctions is large, their interaction is always negative ( repulsion "), hence the mean field approximation can be used. To use this approximation, the « molecular field » $B_{\lambda}$ conjugated to $a_{\lambda}^{2}$ must be introduced :

$$
B_{\lambda}^{i j}=\sum_{\mu} a_{\mu}^{i} a_{\mu}^{j}\left(1-\frac{2}{3} \delta_{\mu \lambda}\right) \sum_{k} \psi_{\mu}^{2}(k) \psi_{\lambda}^{2}(k)=\frac{1}{n} \delta_{i j} B+B_{\lambda}^{i j}
$$

Fluctuations of $B_{\lambda}^{i j}$ can be neglected in the zeroth order approximation, then the MFA equation follows :

$$
\begin{gathered}
\dot{\mathbf{a}}_{\lambda}=-\Gamma_{0} \frac{\delta H_{0}}{\delta \mathbf{a}_{\lambda}}+\zeta_{\lambda}(t) ; \quad H_{0}=\sum_{\lambda} H_{0}^{(\lambda)} \\
H_{0}^{(\lambda)}=\frac{1}{2}\left\{\mathbf{a}_{\lambda}^{2}\left[(\tau+B)^{2}-\varepsilon_{\lambda}\right]+C_{n}\left(\mathbf{a}_{\lambda}^{2}\right)^{2} \sum_{\lambda} \psi_{\lambda}^{4}(i)\right\}
\end{gathered}
$$

where $C_{n}=(n-1) / 3(n+2)$ if $n \geqslant 2, C_{1} \simeq|\tau|$.

The term in the last equation is negative for the filled modes $\lambda$. The effect of thermal fluctuations on the value $\mathbf{a}_{\lambda}^{2}$ is neglegible in the equilibrium temperature range $-\tau \gg \tau_{0}$. Therefore, $\mathbf{a}_{\lambda}^{2}$ can be found from the equation $\delta H_{0} / \delta \mathbf{a}_{\lambda}=0$. Substituting it into(10) we get :

$$
(\tau+B)^{2}-\varepsilon_{\mathrm{c}}=\zeta \varepsilon_{0} ; \quad \zeta=\varepsilon_{0} / \tau^{2}(n=1), \varepsilon_{0} /|\tau|(n \geqslant 2) ; \quad \varepsilon_{0}=\tau_{0}^{2} \simeq Z^{-4 / 3} .
$$

Equations (11) and (12) govern the spin relaxation in the temperature range $\tau_{0} \ll-\tau \ll \tau_{1}$ $\left(\tau_{1} \simeq Z^{-\rho}, \rho=1 / 3\right.$ for $n=1, \rho=4 / 15$ for $n \geqslant 2$ ). The $a_{\lambda}$ sign interaction becomes important at temperatures $\tau \sim-\tau_{1}$. We have shown $[2,3]$ that the equilibrium properties of the obtained system of interacting amplitude signs are the same as the properties of the initial model (1) to (3) with renormalized values of $T$, and $Z$. This statement is also valid for non-equilibrium properties, but there exists an additional renormalized parameter $\Gamma$ (the rate of isolated spin relaxation). The renormalized value of $\Gamma$ follows from equation (11).

Henceforth we consider the cases $n \geqslant 2, n=1$ separately. In the first case the changes of the absolute value of $a_{\lambda}$ can be neglected; only fluctuations of their directions must be taken into account. The transformation of variables $\mathbf{a}_{\lambda} \rightarrow \sigma_{\lambda}$ leads to :

$$
\Gamma_{1}=\Gamma_{0} a_{\lambda}^{-2} \simeq \Gamma_{0} Z_{0}^{-2}\left(\tau_{0} / \tau_{1}\right)^{2} \simeq Z^{-14 / 5} \cdot \Gamma_{0} .
$$

While deriving the second equality of (13) we have used the value of $a_{\lambda_{0}}^{2}$ for the characteristic filled modes, i.e. modes with $\varepsilon_{\lambda}-\varepsilon_{0} \sim \zeta \varepsilon_{0}$ (these modes are responsible for direction correlation at $\tau \sim-\tau_{1}$ ). Note that the sign $\simeq$ in equation (13) and others mean equality up to unknown numerical factors.

In the Ising spin case the change of sign is possible only due to thermally activated motion of $a_{\lambda}$ over the barrier in the $a_{\lambda}^{2} \ll a_{\lambda_{0}}^{2}$ region. Substitution of the value of $a_{\lambda_{0}}^{2}$ into $H_{0}^{(\lambda)}$ yields the barrier energy :

$$
\Delta H^{(\lambda)}=\frac{1}{4} a_{\lambda_{0}}^{2}\left[(\tau+B)^{2}-\varepsilon_{\lambda}\right] \simeq \frac{|\tau|}{\tau_{0}}
$$


We see that the barrier energy $\Delta H^{(\lambda)} \gg T$ at $-\tau \gg \tau_{0}$ (remember that $T \approx T_{0}=1$ ), therefore, the $\sigma_{\lambda}$ sign changes occur very seldom. The rate of these changes can be easily estimated from the usual Kramers formula applied to the $H_{0}^{(\lambda)}$ potential (11). At $|\tau| \sim \tau_{1}$ (i.e. when the "spin " interaction is of the order of unity) we obtain :

$$
\Gamma_{1}^{-1} \simeq \Gamma_{0}^{-1} Z_{0}^{2} \exp \left(Z_{0}^{1 / 3}\right) .
$$

It is the value of $\Gamma_{1}$ that will be the new one-spin relaxation rate in the Glauber equations (5) for the « spins " $\sigma_{\lambda}$. Now we may repeat once more all the procedure described above with initial values of $\Gamma_{1}, Z_{1}$, and obtain $\Gamma_{2}, Z_{2}$ and so on.

4. - If the temperature $T$ decreases down to the freezing point $T_{\mathrm{f}}$ the number of hierarchy levels and the maximum relaxation time increase. The maximum relaxation time is equal to $\Gamma_{N}^{-1}(N$ th level relaxation time) at such a real temperature $T_{N}^{*}$ that the effective temperature of the $N$ th level $\theta^{(N)}$ is of the order of the " transition » temperature $\theta_{0}^{(N)}$ of this level $\left(\right.$ e.g. $\left.\theta^{(0)}=1\right)$. The values of $\Gamma_{N}$ can be determined by the recurrent equations following from (13) and (15) :

$$
\begin{gathered}
-\ln \Gamma_{N+1}=-\ln \Gamma_{N}+\frac{14}{5} \ln Z_{N} \quad(n \geqslant 2) \\
-\ln \Gamma_{N+1}=-\ln \Gamma_{N}+Z_{N}^{1 / 3}+2 \ln Z_{N} \quad(n=1)
\end{gathered}
$$

The solutions of (16) and (17) are evident :

$$
\begin{gathered}
\Gamma_{N}=\Gamma_{0} \exp \left\{-\frac{14}{5} \sum_{m=0}^{N-1} \ln Z_{m}\right\}(n \geqslant 2) \\
\Gamma_{N}=\Gamma_{0} \exp \left\{-2 \sum_{m=0}^{N-1} \ln Z_{m}\right\} \exp \left\{-\sum_{m=0}^{N-1} Z_{m}^{1 / 3}\right\} .
\end{gathered}
$$

The values of $Z_{N}$ and $T_{N}^{*}-T_{\mathrm{f}}$ have been obtained in [2, 3] :

$$
\ln Z_{N} \simeq q^{N} \ln Z_{0}
$$

where $q=\frac{4}{3}$ for $n=1, q=\frac{32}{15}$ for $n \geqslant 2$ and

$$
-\ln \frac{T_{N}^{*}-T_{\mathrm{f}}}{T_{\mathrm{f}}}=\frac{p}{q-1}\left(q^{N}-1\right) \ln Z_{0}
$$

where $p=1 / 3$ for $n=1, p=4 / 15$ for $n \geqslant 2\left({ }^{1}\right)$. Substitution of equations (20) into (18) and (19) yields :

$$
\Gamma_{N}=\Gamma_{0} \exp \left\{-\frac{\dot{4} 2}{17}\left[\left(\frac{32}{15}\right)^{N}-1\right] \ln Z_{0}\right\}
$$

for vector spins and

$$
\Gamma_{N}=\Gamma_{0} \exp \left\{-6\left[\left(\frac{4}{3}\right)^{N}-1\right] \ln Z_{0}\right\} \exp \left\{-Z_{0}^{\left[(4 / 3)^{N / 4}\right]} F(N)\right\} .
$$

$\left({ }^{1}\right)$ The difference between the exponents in the cases of vector and Ising spins results from the different behaviour of amplitudes $a_{\lambda}(\tau)$ (cf. (11), (12)), (for further details see [3]). 
The $F(N)$ factor in (23) can be represented in the convenient form :

$$
F(N)=\sum_{m=0}^{N-1} Z_{N-1}^{1 / 3\left[(3 / 4)^{m-1}\right]}
$$

If $N \rightarrow \infty$ then $Z_{N-1} \rightarrow \infty$ and $F(N)$ tend to unity, but very slowly : it can be approximated $F(N) \simeq 1$ only for $Z_{N-1} \gtrsim 10^{4}$, at smaller $Z_{N-1}$ we use rough approximation $F(N) \sim$ $10\left(\frac{3}{4}\right)^{N} / \ln Z_{0}$. Inserting (21) into (22), (23) we get the value of the maximum relaxation time at temperatures $T=T_{N}^{*}$ :

$$
\begin{gathered}
t_{\max } \simeq\left(T_{\mathrm{f}} / T-T_{\mathrm{f}}\right)^{21 / 2} ; n \geqslant 2 \\
t_{\max } \simeq\left(\frac{T_{\mathrm{f}}}{T-T_{\mathrm{f}}}\right)^{6} \exp \left\{\left(\frac{Z_{0} T_{\mathrm{f}}}{T-T_{\mathrm{f}}}\right)^{1 / 4} F(N)\right\} ; n=1 .
\end{gathered}
$$

As in the case of the non-linear equilibrium susceptibility $[2,3]\left({ }^{2}\right)$ formulae $(22)$ and $(23)$ are a poor approximation for $t_{\max }(\tau)$ in the whole $\tau$-range, and represent rather the upper envelope of this function. Note that formulae (25) and (26) hold only for the close vicinity of the freezing temperature point where a lot of hierarchy levels are significant. In the time region $t \ll t_{\max }$, the relaxation is not exponential. Let us estimate this time dependence : all hierarchy levels up to the $N$ th level relax within the time $t \ll t_{\max }$, where $N$ can be determined if $t=\Gamma_{N}^{-1}$ is inserted into equations (22) and (23). Now estimate the part ( $W_{N}$ of the observed spin which relaxes together with the $N$ th hierarchy level, i.e. the part of the system magnetic moment which is governed by the $N$ th level variables. For this reason we determine the total moment, governed by one $N$ th level mode, multiplied by the number of these modes and divided by the total number of spins, which yields :

$$
\begin{aligned}
& W_{N}=\exp \left[-\frac{9}{2}\left(\left(\frac{4}{3}\right)^{N}-1\right) \ln Z_{0}\right]=\exp \left[-\frac{9}{2}\left(\ln Z_{N}-\ln Z_{0}\right)\right](n=1) \\
& W_{N}=\exp \left[-\frac{25}{17}\left(\ln Z_{N}-\ln Z_{0}\right)\right](n \geqslant 2) .
\end{aligned}
$$

Substituting the value of $N(t)$ derived from (22) and (23) into(27) we get the remaining (at time $t$ ) value of the magnetic moment :

$$
M(t) \sim\left(\Gamma_{0} t\right)^{-25 / 42} \quad \text { (vector spins) }
$$

In the Ising case there are two regimes, depending on the relative value of the first and second factors in formula (26). At not too large times the first factor is the most important, so we get :

$$
M(t) \sim\left(\Gamma_{0} t\right)^{-3 / 4}
$$

In the opposite limit :

$$
M(t) \sim\left(\ln \Gamma_{0} t\right)^{-18} .
$$

The quantity $M(t)$ in (28)-(30) is the correlation function $M(t)=\overline{\langle S(0) S(t)\rangle}$; it coincides also with the response to the step-like magnetic field $h(t)=h_{0} \theta(-t)$. This coincidence follows from

(2) We want to correct the numerical error in [2] : the upper envelope is $\tilde{\chi}_{+}(T) \sim\left(T-T_{\mathrm{f}}\right)^{-6}$ instead of $\left(T-T_{\mathrm{f}}\right)^{-5}$. In the vector spin case we obtain $\tilde{\chi}_{+} \sim\left(T-T_{\mathrm{f}}\right)^{-19 / 2}, \tilde{\chi}_{-.} \sim\left(T-T_{\mathrm{f}}\right)^{-5}[3]$. 
the equality $\chi(t)=-\partial M / \partial t$ (being a consequence of FDT-theorem). Equations (28) to (30) are valid if $t \Gamma_{0} \gg 1$, i.e. many hierarchy levels do participate in the relaxation.

5. - Now we discuss the problem of the stability of the vector model solution with respect to the weak isotropy breaking due to weak random or homogeneous anisotropy which are always present in the real system (e.g. the Bzyaloshinsky-Moria [7] interaction leads to random anisotropy). The anisotropy leads to the appearance of the additional terms in the free energy :

$$
\begin{gathered}
\delta F=-\sum_{i} \Lambda\left(\sigma_{i}^{x}\right)^{2}+\sum_{i j} G_{i j}^{\alpha \beta} \sigma_{i}^{\alpha} \sigma_{j}^{\beta} \\
\overline{G_{i j}^{\alpha \beta} G_{i^{\prime} j^{\prime}}^{\alpha^{\prime} \beta^{\prime}}}=\frac{1}{4}\left(\delta_{i i^{\prime}} \delta_{j j^{\prime}}+\delta_{i j^{\prime}} \delta_{j i^{\prime}}\right)\left(\delta_{\alpha \alpha^{\prime}} \delta_{\beta \beta^{\prime}}+\delta_{\alpha \beta^{\prime}} \delta_{\beta \alpha^{\prime}}\right) G^{2} .
\end{gathered}
$$

The additional terms in the initial free energy bring about analogous terms in the effective free energy of the next hierarchy level :

$$
\delta \tilde{F}=-\tilde{\Lambda} \sum_{\lambda}\left(\sigma_{\lambda}^{x}\right)^{\dot{2}}+\sum_{\lambda \mu} \tilde{G}_{\lambda \mu}^{\alpha \beta} \sigma_{\lambda}^{\alpha} \sigma_{\mu}^{\beta}
$$

Let us express the new anisotropy constants $\tilde{\Lambda}, \tilde{G}$, using formulae for the variables of the next hierarchy level. We get :

$$
\begin{aligned}
& \tilde{\Lambda}=Z^{14 / 5} \Lambda \\
& \tilde{G}=Z^{7 / 10} G .
\end{aligned}
$$

Thus we see that the effective anisotropy increases rapidly with increasing number of hierarchy levels, i.e. the vector model is unstable with respect to symmetry breaking. Note that both types of anisotropy lead to the same asymptotic behaviour as that of the Ising spins. This has been proved for the model with infinite range interaction in [8]. In our case it follows from the fact that the eigenfunctions of the exchange matrix $\mathfrak{J}_{i j} \delta^{\alpha \beta}+G_{i j}^{\alpha \beta}$ are non-degenerate, hence the eigenfunctions $\psi_{\lambda}^{\alpha}(i)$ have a vector index $\alpha$, whereas the amplitudes $a_{\lambda}$ of the next level have none.

6. - We have shown that the hierarchical structure of spin modes, appearing in the vicinity of $T_{\mathrm{f}}$, leads to a sharp slowing down of spin relaxation; the relaxation time spectrum expands, the maximum time is determined by (25) and (26). In the intermediate time region the magnetization relaxation is given by formulae (28) to (30). It is interesting that the obtained relaxation law (28) in the case of vector spins is rather close to the result of the mean field theory $M(t) \sim t^{-1 / 2}[9]$. We emphasize here that the numerical values of the exponents in formulae (25), (26), and (28)-(30) are based on the hypotheses of the localization theory. In the spin glasses with weak anisotropy (31) one should expect the crossover from the vector regime to the Ising regime, as $T-T_{\mathrm{f}}$ decreases.

Qualitatively the analogous scheme of the hierarchical dynamics of glasses has been recently investigated in [4]. Some versions of the $W_{N}$ and $\Gamma_{N}$ values behaviour, depending on the number $N$, have been suggested in this paper. In this Letter we have tried to obtain the $W_{N}$ and $\Gamma_{N}$ dependences, employing the microscopic model of spin glass. The obtained dependences do not coincide with any of the versions of the paper [4]. An important qualitative difference is the growth of $\ln \left(\Gamma_{N} / \Gamma_{N-1}\right)=\mu_{N}$ (in the notations of [4]). Note, that the calculations of the work [4] give the saddle point value of $\mu_{n} \simeq\left[t / \tau_{0} \exp \left(\mu_{0} \gamma_{0}\right)\right]^{-\beta}\left(\beta^{-1}-1\right)$ therefore, in order to obtain the Kohlrausch law one must consider $\mu_{n} \ll 1$ (" weak constraint »), having an obscure physical meaning. The same qualitative ideas have also been expressed by Hertz. Another important difference is the finite number of levels at $T>T_{\mathrm{f}}$. 
In conclusion, let us compare our results with the experiment. As a rule, the $t_{\max }(T)$ dependences are interpreted either by the scaling formulae $t_{\max } \sim\left(T-T_{\mathrm{f}}\right)^{-v z}$, or by the Vogel-Fulcher law $t_{\max } \sim \exp \left(E / T-T_{\mathrm{f}}\right)$, the first description is possible at relatively fast observation times (see [11] for example, where $t \approx 10^{-9} \mathrm{~s}$ ) and the second, for quasistatic measurements at $t \approx 10^{-2}$ $10^{4} \mathrm{~s}$, see $[12,13]$. It seems to us that the formulae $t_{\max } \sim\left(T-T_{\mathrm{f}}\right)^{-v z} \exp \left(c\left(T-T_{\mathrm{f}}\right)^{-\omega}\right)$ are worth being used in the whole time interval for the experimental description. Moreover it seems plausible that the frequency decrease from the HF measurement $\left(f \sim 10^{9} \mathrm{~s}^{-1}\right)$ to the LF measurement $\left(f \sim 10^{-4}-10^{2} \mathrm{~s}^{-1}\right)$ is accompanied with the crossover from the " vector » regime (25) to the Ising regime (26).

We believe that there exist real spin glasses, corresponding literally to the model investigated here. These are rare-earth alloys $Y_{1-X} R_{X}(R=E r, D y, T b, G d)[14,15]$ with a low $X$ (which is probably in the range $X \sim 0.5-2 \%$ ). At higher concentrations $X \sim 2-5 \%$ the local helical order has been observed in these alloys [14] at low temperatures (the theory of this state has been proposed in [16]). This means that the interaction radius is large : $R_{0} \gg a \sim p_{0}^{-1}$ ( $a$ is the lattice constant, and $p_{0}$, the helix wave vector). So there must exist such a concentration range [16], that the low temperature phase no longer possesses the local helical order, but the effective number of neighbours $(Z)$ is still large.

\section{Acknowledgments.}

We are grateful to A. I. Larkin for useful discussions.

\section{References}

[1] Edwards, S. F., ANDerson, P. W., J. Phys. F 5 (1975) 965.

[2] Feigel'man, M. V., IofFe, L. B., J. Physique Lett. 45 (1984) L-475.

[3] IOFFE, L. B., Ph. D. thesis ; IOFFE, L. B., FeIGel'man, M. V., ZhETF 89 (1985) N2.

[4] Palmer, R. G., Stein, D. L., Abrahams, E., Anderson, P. W., Phys. Rev. Lett. 53 (1984) 958.

[5] Thouless, D. J., Anderson, P. W., Palmer, R. G., Philos. Mag. 35 (1977) 593.

[6] Bray, A. J., MoOre, M. A., J. Phys. C 14 (1981) 2629.

[7] Levy, P. M., Fert, A., Phys. Rev. B 23 (1981) 4667.

[8] Kotliar, G., Sompolinsky, H., Phys. Rev. Lett. 53 (1984) 1751.

[9] Sompolinsky, H., Zippelius, A., J. Phys. C 15 (1982) L1059.

[10] HertZ, J. A., NORDITA, preprint 84/33 (1984).

[11] WU WeI-YU, MozUrkevich, G., ORBACH, R., preprint (1984).

[12] Wenger, L. E., in Heidelberg Colloquium on Spin glasses, Eds. J. L. van Hemmen and I. Morgenstern, Lecture Notes in Physics 192 (Springer Verlag) 1983.

[13] Lundgren, L., Svendlinh, P., Beckman, O., Phys. Rev. B 26 (1982) 3990.

[14] Babershke, K. et al., Phys. Rev. B 29 (1984) 4999. Bouchiat, H., MaIlly, D., preprint (1984).

[15] Wenger, L. E., Mydosh, J. A., J. Appl. Phys. 55 (1984) 1850.

BONJOUR, E. et al., in Proc. of LT-17 (1984) p. 643.

[16] IofFe, L. B., Feigel'man, M. V., ZhETF 88 (1985) N2. 